Interior-point solver for convex separable block-angular problems

Jordi Castro

Dept. of Statistics and Operations Research

Univ. Politècnica de Catalunya

jordi.castro@upc.edu

Research Report UPC-DEIO DR 2014-03

Report available from http://www-eio.upc.es/ j jcastro 



\title{
Interior-point solver for convex separable block-angular problems
}

\author{
Jordi Castro* \\ Dept. of Statistics and Operations Research, Universitat Politècnica de Catalunya, Jordi Girona \\ 1-3, 08034 Barcelona
}

\begin{abstract}
Constraints matrices with block-angular structures are pervasive in Optimization. Interior-point methods have shown to be competitive for these structured problems by exploiting the linear algebra. One of these approaches solved the normal equations using sparse Cholesky factorizations for the block constraints, and a preconditioned conjugate gradient (PCG) for the linking constraints. The preconditioner is based on a power series expansion which approximates the inverse of the matrix of the linking constraints system. In this work we present an efficient solver based on this algorithm. Some of its features are: it solves linearly constrained convex separable problems (linear, quadratic or nonlinear); both Newton and second-order predictor-corrector directions can be used, either with the Cholesky+PCG scheme or with a Cholesky factorization of normal equations; the preconditioner may include any number of terms of the power series; for any number of these terms, it estimates the spectral radius of the matrix in the power series (which is instrumental for the quality of the preconditioner). The solver has been hooked to SML, a structure-conveying modelling language based on the popular AMPL modeling language. Computational results are reported for some large and/or difficult instances in the literature: (1) multicommodity flow problems; (2) minimum congestion problems; (3) statistical data protection problems using $\ell_{1}$ and $\ell_{2}$ distances (which are linear and quadratic problems, respectively), and the pseudo-Huber function, a nonlinear approximation to $\ell_{1}$ which improves the preconditioner. In the largest instances, of up to 25 millions of variables and 300000 constraints, this approach is from two to three orders of magnitude faster than state-of-the-art linear and quadratic optimization solvers.
\end{abstract}

Keywords: interior-point methods; structured problems; normal equations; preconditioned conjugate gradient; large-scale optimization; optimization software

AMS Subject Classification: 90C51, 90C25, 90C06, 90C05, 90C20

\section{Introduction}

Block-angular structures are used as a modelling tool in many situations, such as multiperiod or multicommodity problems, two-stage stochastic problems, and, in general, models involving linking variables or linking constraints. These structured problems have applications, for instance, in the energy, logistics, telecommunications and big-data fields. The resulting optimization problems have in common a huge number of variables, and a large number of linear constraints. Interior-point methods (IPMs) are in general competitive against other techniques in those cases.

This work presents an efficient interior-point solver for block-angular convex optimization problems, which relies on a combination of Cholesky factorizations and preconditioned conjugate gradient (PCG) for the solution of the linear systems at each interiorpoint iterations. This Cholesky $+\mathrm{PCG}$ combination was initially suggested for the case of multicommodity flows in [10]; it was later extended to general problems in [12], including a Matlab prototype. The solver introduced in this work, named BlockIP is not merely a "C ++ translation" of this Matlab prototype. It includes many other features,

\footnotetext{
*Email: jordi.castro@upc.edu
} 
being the three most relevant: (i) it may solve convex nonlinear separable optimization problems; (ii) the addition of proximal point and quadratic regularizations, which may significantly improve the quality of the preconditioner as shown in [14]; (ii) an estimation of the spectral radius of a certain matrix which intervenes in the preconditioner, following [9], which can be used as a measure of the quality of preconditioner. This estimation has been extended in this work to more complex preconditioners, i.e., involving more terms of a certain power series; Proposition 3.5 provides this result. In addition, BlockIP resulted to be much more efficient than the Matlab prototype, allowing us to solve problems of up to 25 millions of variables and 300000 constraints (see Section 5), which were out of the scope of [12].

BlockIP solves the normal equations, unlike other PCG-based IPMs that focus on the augmented system, such as [5, 22, 31]. Normal equations were solved by some of the earlier preconditioners for network flows problems [18, 33], but also by some recent approaches [3]. Although PCG usually provides an approximate solution of the linear systems of equations, it has recently been shown that those inexact solutions also guarantee the convergence of the IPM [20].

The structure of the paper is as follows. The formulation of the block-angular problem considered is given in Section 2, Section 3 outlines the PCG-based IPM, including three subsections summarizing some of the main features implemented in the solver, namely, the power series preconditioner; improving the preconditioner by reducing the spectral radius of a certain matrix through quadratic or nonlinear terms in the objective; and the estimation of this spectral radius by Ritz values. Though the material of these three subsections comes, mainly, from [12], [14] and [9], it is included here for completeness. Section 4 describes the specialized solver. Finally, Section 5 provides computational results in the solution of three types of problems: multicommodity flows, minimum congestion, and a disclosure control problem in statistical tabular data.

\section{Convex block-angular problems}

The standard form of the linearly constrained convex block-angular problems considered in this work is

$$
\begin{aligned}
& \min \sum_{i=0}^{k} f_{i}\left(x^{i}\right) \\
& \text { s. to }\left[\begin{array}{ccc}
A_{1} & & \\
& \ddots & \\
& & A_{k} \\
L_{1} & \ldots & L_{k} \\
& I
\end{array}\right]\left[\begin{array}{c}
x^{1} \\
\vdots \\
x^{k} \\
x^{0}
\end{array}\right]=\left[\begin{array}{c}
b^{1} \\
\vdots \\
b^{k} \\
b^{0}
\end{array}\right] \\
& 0 \leq x^{i} \leq u^{i} \quad i=0, \ldots, k .
\end{aligned}
$$

Matrices $A_{i} \in \mathbb{R}^{m_{i} \times n_{i}}$ and $L_{i} \in \mathbb{R}^{l \times n_{i}}, i=1, \ldots, k$ define the block and linking constraints, respectively, $k$ being the number of blocks. Vectors $x^{i} \in \mathbb{R}^{n_{i}}, i=1, \ldots, k$, are the variables for each block. $x^{0} \in \mathbb{R}^{l}$ are the slacks of the linking constraints. $b^{i} \in \mathbb{R}^{m_{i}}, i=1, \ldots, k$ is the right-hand-side vector for each block of constraints, whereas $b^{0} \in \mathbb{R}^{l}$ is for the linking constraints. The upper bounds for each group of variables are defined by $u^{i}, i=0, \ldots, k$. Note that with this standard formulation linking constraints are of the form $b^{0}-u^{0} \leq \sum_{i=1}^{k} L_{i} x^{i} \leq b^{0}$. As it will be shown later, slacks of linking constraints play a significant role in the quality of the preconditioner, and they should not 
be removed. Equality linking constraints can be formulated by setting $u^{0} \approx 0$. Functions $f_{i}: \mathbb{R}^{n_{i}} \rightarrow \mathbb{R}, i=0, \ldots, k$, are assumed to be convex. Although the specialized IPM to be described is valid for any $f_{i}$, for the sake of efficiency we will restrict to separable functions, i.e., $\nabla^{2} f_{i}\left(x^{i}\right)$ are (positive semidefinite) diagonal matrices. Note that any convex quadratic problem can be transformed into a separable equivalent one by the addition of extra variables and constraints [35, Ch.23]. It is also worth to remark that slacks may also intervene in the objective function; this may be helpful to preserve the separability of the problem, avoiding terms of the form $f_{0}\left(b^{0}-\sum_{i=1}^{k} L_{i} x^{i}\right)$. The particular case of linear and convex quadratic separable problems is obtained with $f_{i}\left(x^{i}\right)=c^{i^{\top}} x^{i}$ and $f_{i}\left(x^{i}\right)=c^{i^{\top}} x^{i}+\frac{1}{2} x^{i^{\top}} Q_{i} x^{i}, Q_{i}$ positive semidefinite and diagonal, for $i=1 \ldots, k$. (1) is an optimization problem with $m=\sum_{i=1}^{k} m_{i}+l$ constraints and $n=\sum_{i=1}^{k} n_{i}+l$ variables.

The structure of the constraints matrix of (11) is usually named primal block-angular. More general structures (i.e., such as those of [21]) are obtained if, for some $i, m_{i}$ is 0 , i.e., a group of variables only appears in the linking constraints. For instance, if $m_{k}=0$ we have

$$
\left[\begin{array}{llll}
A_{1} & & & \\
& \ddots & & \\
& & & \\
& & A_{k-1} & \\
L_{1} & \ldots & L_{k-1} & L_{k} I
\end{array}\right]
$$

Dual block-angular structures, as those appearing in two-stage stochastic optimization problems with recourse, can be formulated as (1) by considering the dual problem. Even problems with both linking variables and linking constraints may be transformed to (1) by replicating columns and adding extra linking constraints.

On the other hand, when $k=1$, the specialized method can be applied to any linearly constrained convex problem by considering a partition of the linear constraints, as follows

$$
\begin{array}{ll}
\min & f(x)+f_{0}\left(x^{0}\right) \\
\text { s. to } & A x=b \\
& L x+x^{0}=b^{0} \\
& 0 \leq x \leq u \quad, \quad 0 \leq x^{0} \leq u^{0} .
\end{array}
$$

If $k=1$ and $l=0$, (10) reduces to the standard linearly constrained convex problem $\min f(x)$ s. to $A x=b, 0 \leq x \leq u$.

\section{Overview of the interior-point method for block-angular problems}

Problem (11) can be written as the general convex optimization problem

$$
\begin{array}{ll}
\min & f(x) \\
\text { s. to } & A x=b \\
& 0 \leq x \leq u,
\end{array}
$$

where $x, u \in \mathbb{R}^{n}, A \in \mathbb{R}^{m \times n}, b \in \mathbb{R}^{m}$, and $f: \mathbb{R}^{n} \rightarrow \mathbb{R}$. Vectors $x, u$ and $b$ are made up of the $k+1$ vectors $x^{i}, u^{i}$ and $b^{i}, i=0 \ldots, k$.

By convexity, the first order KKT conditions of (4) are necessary and sufficient. Perturbing its complementarity equations with a parameter $\mu \in \mathbb{R}^{+}$, the KKT- $\mu$ conditions 
can be written as

$$
\begin{aligned}
r_{c} \equiv \nabla f(x)-\left(A^{\top} \lambda+z-w\right) & =0 \\
r_{b} \equiv & b-A x=0 \\
r_{x z} \equiv & =0 \\
r_{s w} \equiv & \mu e-X Z e=0 \\
\mu e-S W e & =0 \\
& (x, s, z, w) \geq 0
\end{aligned}
$$

where $e \in \mathbb{R}^{n}$ is a vector of 1 's; $\lambda \in \mathbb{R}^{m}, z \in \mathbb{R}^{n}$ and $w \in \mathbb{R}^{n}$ are, respectively, the vectors of Lagrange multipliers of the equality constraints, lower and upper bounds; $s=u-x$; and matrices $X, Z, S, W \in \mathbb{R}^{n \times n}$ are diagonal matrices made up of vectors $x, z, s, w$. The set of unique solutions of (5) for each $\mu$ value is known as the central path, and when $\mu \rightarrow 0$ these solutions converge to those of (44). The nonlinear system (5) is usually solved by a sequence of damped Newton's directions (i.e., with step length reduction to preserve the nonnegativity of variables), reducing the $\mu$ parameter at each iteration. This procedure is known as the primal-dual path-following interior-point algorithm. An excellent discussion about the theoretical properties of this and other interior-point algorithms can be found in [29, 34, 36].

The linearization of (5) provides a linear system of variables $\Delta x, \Delta \lambda, \Delta z$ and $\Delta w$. After eliminating $\Delta w$ and $\Delta z$, as follows:

$$
\begin{gathered}
\Delta z=X^{-1} r_{x z}-X^{-1} Z \Delta x \\
\Delta w=S^{-1} r_{s w}+S^{-1} W \Delta x,
\end{gathered}
$$

we obtain the augmented system form

$$
\left[\begin{array}{cc}
-\Theta^{-1} & A^{\top} \\
A &
\end{array}\right]\left[\begin{array}{c}
\Delta x \\
\Delta \lambda
\end{array}\right]=\left[\begin{array}{c}
r \\
r_{b}
\end{array}\right],
$$

where $\Theta$ and $r$ are defined as

$$
\Theta=\left(Z X^{-1}+W S^{-1}+\nabla^{2} f(x)\right)^{-1} \quad r=r_{c}+S^{-1} r_{s w}-X^{-1} r_{x z}
$$

Note that, if the objective function is separable, $\Theta$ is an easily computable diagonal matrix. Additionally, eliminating $\Delta x$ from the first group of equations of (8), the normal equations are obtained:

$$
\begin{aligned}
\left(A \Theta A^{\top}\right) \Delta \lambda & =r_{b}+A \Theta r \\
\Delta x & =\Theta\left(A^{\top} \Delta \lambda-r\right) .
\end{aligned}
$$

The method of this paper solves the normal equations, and thus the Newton direction is computed by (10), (111), (6) and (7).

Exploiting the structure of $A$, and appropriately partitioning $\Theta$, as follows

$$
A=\left[\begin{array}{ccc}
A_{1} & & \\
& \ddots & \\
& & A_{k} \\
L_{1} & \ldots & L_{k} I
\end{array}\right] \quad \Theta=\left[\begin{array}{cccc}
\Theta_{1} & & & \\
& \ddots & & \\
& & \Theta_{k} & \\
& & & \Theta_{0}
\end{array}\right]
$$


the matrix of system (10) can be recast as

$$
A \Theta A^{\top}=\left[\begin{array}{cccc|c}
A_{1} \Theta_{1} A_{1}^{\top} & & & & A_{1} \Theta_{1} L_{1}^{\top} \\
& \ddots & & \\
& & A_{k} \Theta_{k} A_{k}^{\top} & A_{k} \Theta_{k} L_{k}^{\top} \\
& & \\
& & \\
& & & \\
& & & \\
L_{1} \Theta_{1} A_{1}^{\top} & \ldots & L_{k} \Theta_{k} A_{k}^{\top} & \Theta_{0}+\sum_{i=1}^{k} & L_{i} \Theta_{i} L_{i}^{\top}
\end{array}\right]=\left[\begin{array}{cc}
B & C \\
C^{\top} & D
\end{array}\right],
$$

$B \in \mathbb{R}^{\tilde{n} \times \tilde{n}}\left(\tilde{n}=\sum_{i=1}^{k} n_{i}\right), C \in \mathbb{R}^{\tilde{n} \times l}$ and $D \in \mathbb{R}^{l \times l}$ being the blocks of $A \Theta A^{\top}$, and $\Theta_{i}$, $i=0, \ldots, k$, the submatrices of $\Theta$ associated with the $k+1$ groups of variables in (11), i.e., $\Theta_{i}=\left(Z_{i} X_{i}^{-1}+W_{i} S_{i}^{-1}+\nabla^{2} f_{i}\left(x^{i}\right)\right)^{-1}$. Denoting by $g$ the right-hand-side of (10), and appropriately partitioning $g$ and $\Delta \lambda$, the normal equations can be written as

$$
\left[\begin{array}{cc}
B & C \\
C^{T} & D
\end{array}\right]\left[\begin{array}{l}
\Delta \lambda_{1} \\
\Delta \lambda_{2}
\end{array}\right]=\left[\begin{array}{l}
g_{1} \\
g_{2}
\end{array}\right]
$$

\subsection{Solving the normal equations by PCG}

Eliminating $\Delta \lambda_{1}$ from the first group of equations of (13), we obtain

$$
\begin{aligned}
\left(D-C^{\top} B^{-1} C\right) \Delta \lambda_{2} & =\left(g_{2}-C^{\top} B^{-1} g_{1}\right) \\
B \Delta \lambda_{1} & =\left(g_{1}-C \Delta y_{2}\right) .
\end{aligned}
$$

System (15) is solved by performing $k$ Cholesky factorizations, one for each diagonal block $A_{i} \Theta_{i} A_{i}^{\top}, i=1 \ldots k$, of $B$. System (14) with the Schur complement

$$
S=D-C^{\top} B^{-1} C
$$

of dimension $l$-the number of linking constraints - , may exhibit a big fill-in, and it is prohibitive if computed by Cholesky. It will be solved by a PCG, which is outlined in Figure 1. A good preconditioner is instrumental. The solver in this work considers the preconditioner derived in [10] for multicommodity flows, and extended to general problems in [12]. The preconditioner relies on the fact that (16) is a P-regular splitting, i.e., $S$ is symmetric and positive definite, $D$ is nonsingular and $D+C^{\top} B^{-1} C$ is positive definite. Therefore the P-regular splitting theorem [4] [32, pp. 254-255] guarantees that

$$
\rho\left(D^{-1}\left(C^{\top} B^{-1} C\right)\right)<1,
$$

where $\rho(\cdot)$ denotes the spectral radius of a matrix (i.e., the maximum absolute eigenvalue). (To simplify the notation, $\rho\left(D^{-1}\left(C^{\top} B^{-1} C\right)\right.$ will be referred as to simply $\rho$.) This allows us to compute the inverse of $S$, as shown by the next result (see [10, Prop. 4] for a proof).

Proposition 3.1 The inverse of the Schur complement $D-C^{\top} B^{-1} C$ can be computed as

$$
\left(D-C^{\top} B^{-1} C\right)^{-1}=\left(\sum_{i=0}^{\infty}\left(D^{-1}\left(C^{\top} B^{-1} C\right)\right)^{i}\right) D^{-1} .
$$


1. Algorithm $P C G\left(S, M, \bar{g}, \Delta \lambda_{2_{0}}, \epsilon, i_{\max }\right)$

2. // Solve $S \Delta \lambda_{2}=\bar{g}$ by PCG with preconditioner $M$

3. Initializations: $i:=0 ; r_{0}:=\bar{g}-S \Delta \lambda_{2_{0}}$;

4. Solve $M z_{0}=r_{0} ; p_{0}:=z_{0}$;

5. while $\left\|r_{k}\right\|>\epsilon$ and $i<i_{\max }$ do

6. $q_{i}:=S p_{i}$;

7. $\alpha_{i}:=\left(z_{i}^{\top} r_{i}\right) /\left(p_{i}^{\top} q_{i}\right)$;

8. $\Delta \lambda_{2_{i+1}}:=\Delta \lambda_{2_{i}}+\alpha_{i} p_{i}$;

9. $\quad r_{i+1}:=r_{i}-\alpha_{i} q_{i}$;

10. Solve $M z_{i+1}=r_{i+1}$;

11. $\quad \beta_{i}:=\left(z_{i+1}^{\top} r_{i+1}\right) /\left(z_{i}^{\top} r_{i}\right)$;

12. $p_{i+1}:=z_{i+1}+\beta_{i} p_{i}$

13. $i:=i+1$;

14. end while

15. Return $\Delta \lambda_{2}:=\Delta \lambda_{2 i}$;

16. End algorithm

Figure 1. The PCG algorithm for the solution of $S \Delta \lambda_{2}=\bar{g} \equiv g_{2}-C^{\top} B^{-1} g_{1}$ with preconditioner $M$

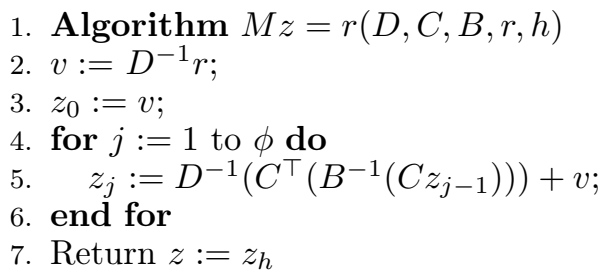

Figure 2. Algorithm for computing $z=M^{-1} r$

The preconditioner $M^{-1}$ is an approximation of $S^{-1}$ obtained by truncating the infinite power series (18) at some term $\phi$. For instance, for $\phi=0$ and $\phi=1$ we have

$$
\begin{array}{lr}
M^{-1}=D^{-1} & \text { if } \phi=0, \\
M^{-1}=\left(I+D^{-1}\left(C^{\top} B^{-1} C\right)\right) D^{-1} & \text { if } \phi=1 .
\end{array}
$$

The larger $\phi$, the better the approximation of the inverse. On the other hand, systems $M z=r$ (for some vectors $z$ and $r$ ) have to be solved at each PCG iteration (step 10 of PCG algorithm of Figure 11), and any extra term in the series means an additional system with matrix $B$. This is clearly seen in the algorithm of Figure 2, which shows how $M z=r$ is iteratively computed in the specialized interior-point solver. Note that matrix $C^{\top} B^{-1} C$ does not need to be built, and aside from the solution of systems with $B$ and $D$, only matrix-vector products with $C$ and $C^{\top}$ (i.e., with $L_{i}, L_{i}^{\top}, A_{i}$ and $A_{i}^{\top}$, $i=1, \ldots, k)$ are required. This also applies to the PCG algorithm of Figure 1] It is thus possible to partially apply the matrix-free paradigm [19]. Efficient implementations of this matrix-vector products for particular $A_{i}$ and $L_{i}, i=1, \ldots, k$, matrices can significantly speed the computational efficiency. Aside from general matrices (either stored rowwise or columnwise), the solver of this paper includes matrix-vector routines for some particular classes of matrices, such as node-arc incidence matrices (both for oriented and nonoriented flows), diagonal and identity matrices, and diagonal-diagonal (i.e., $\left[D_{1} D_{2}\right], D_{1}, D_{2}$ being diagonal) and identity-identity matrices (i.e., $[I I], I$ being the identity matrix). Other matrix types can be easily added.

The value of $\phi$ that optimizes the tradeoff between a good quality and an efficient preconditioner is problem dependent, and finding a dynamic updating procedure for each interior-point iteration is still work in progress. It is worth noting that, although Proposition 3.1 is valid for any primal block-angular problem, the preconditioner is only useful in practice for separable problems; otherwise, nondiagonal $\Theta_{i}$ matrices make systems with $B$ prohibitive. 


\subsection{Improving the spectral radius}

The quality of the preconditioner depends on $\rho$, which is always in $[0,1)$ : the farther from 1 , the closer $M^{-1}$ is to $S^{-1}$. In practice it has been observed that $\rho$ comes closer to 1 as we approach the optimal solution for most instances. However, as shown in [14], non-zero Hessians reduce $\rho$, and this opens the possibility for improving the preconditioner by the addition of a quadratic regularization term. This assertion is supported by the following theorem and proposition (see [14] for a proof):

Theorem 3.2 The spectral radius $\rho$ of $D^{-1}\left(C^{\top} B^{-1} C\right)$ is bounded by

$$
0 \leq \rho \leq \max _{j \in\{1, \ldots, l\}} \frac{\gamma_{j}}{\left(\frac{u_{j}}{v_{j}}\right)^{2} \Theta_{0 j}+\gamma_{j}}<1
$$

where $u$ is the eigenvector (or one of the eigenvectors) of $D^{-1}\left(C^{\top} B^{-1} C\right)$ for $\rho ; \gamma_{j}, j=$ $1, \ldots, l$, and $V=\left[V_{1} \ldots V_{l}\right]$, are respectively the eigenvalues and matrix of columnwise eigenvectors of $\sum_{i=1}^{k} L_{i} \Theta_{i} L_{i}^{\top} ; v=V^{\top} u$; and, abusing of notation, we assume that for $v_{j}=0,\left(u_{j} / v_{j}\right)^{2}=+\infty$.

Proposition 3.3 Consider a linear problem and a non-linear one obtained by adding (likely small) Hessian terms $\nabla^{2} f_{i}\left(x^{i}\right) \succ 0, i=1, \ldots, k$. Assume $\hat{u}_{j} / \hat{v}_{j} \leq u_{j} / v_{j}, j=$ $1, \ldots, l$, where "hatted" and "non-hatted" terms refer, respectively, to the linear and nonlinear problems, and $u$ and $v$ are defined as in Theorem 3.2. Then bound (19) is smaller for the non-linear than for the linear problem.

The fulfillment of the technical condition $\hat{u}_{j} / \hat{v}_{j} \leq u_{j} / v_{j}, j=1, \ldots, l$ in Proposition 3.3 is problem dependent, and it may not be easy to check. However for some important classes of problems, such as those where $L_{i}, i=1, \ldots, k$, are diagonal matrices, this assumption may be seen to hold [14]. It is also worth to note that the presence of slacks in the linking constraints is instrumental, otherwise $\Theta_{0}=0$ and the bound (19) would be equal to 1 .

Theorem 3.2 and Proposition 3.3 state that the bound on the spectral radius (and thus eventually the spectral radius when it is close to 1 ) is reduced if we consider a Hessian term to $\Theta$, defined in (9). In the limit, as shown by next proposition (see [14] for a proof), the spectral radius goes to 0 for very large Hessians, and therefore PCG will become extremely efficient:

Proposition 3.4

$$
\lim _{\substack{\nabla^{2} f_{i}\left(x^{i}\right) \rightarrow+\infty \\ i=1, \ldots, k}} \rho=0
$$

In practice, if the problem is linear or the Hessian is close to 0 , a non-zero Hessian can be added by a quadratic regularization. The interior-point solver of this work implements two types of regularizations: a proximal point and a quadratic regularization. They are based on the addition of a quadratic term to the standard logarithmic barrier function $B(x, \mu)$ of (4)

$$
B(x, \mu) \triangleq f(x)+\mu\left(-\sum_{i=1}^{n} \ln x_{i}-\sum_{i=1}^{n} \ln \left(u_{i}-x_{i}\right)\right),
$$


$\mu \in \mathbb{R}^{+}$being the barrier parameter. In the proximal point regularization, $B(x, \mu)$ is replaced by

$$
B_{P}(x, \mu) \triangleq f(x)+\frac{1}{2}(x-\bar{x})^{\top} Q_{P}(x-\bar{x})+\mu\left(-\sum_{i=1}^{n} \ln x_{i}-\sum_{i=1}^{n} \ln \left(u_{i}-x_{i}\right)\right),
$$

$Q_{P}$ being a diagonal positive definite matrix (which can be dynamically updated at each interior-point iteration, as done in [1]), and $\bar{x}$ the current point obtained by the interiorpoint algorithm. The alternative quadratic regularization introduced in [14] considers

$$
B_{Q}(x, \mu) \triangleq f(x)+\mu\left(\frac{1}{2} x^{\top} Q_{R} x-\sum_{i=1}^{n} \ln x_{i}-\sum_{i=1}^{n} \ln \left(u_{i}-x_{i}\right)\right),
$$

$Q_{R}$ being a diagonal positive semidefinite matrix. Unlike $B_{P}, B_{Q}$ does not depend on the current point, and its reduction to 0 is controlled by $\mu$, the standard barrier parameter.

Using either $B, B_{P}$, or $B_{Q}$ only changes the dual feasibility of KKT conditions and matrix $\Theta$, defined in (5) and (9), respectively. Dual feasibility becomes

$$
\begin{array}{ll}
A^{\top} \lambda+z-w=\nabla f(x) & \text { for } B, \\
A^{\top} \lambda+z-w=\nabla f(x)+Q_{P}(x-\bar{x}) & \text { for } B_{P}, \text { and } \\
A^{\top} \lambda+z-w=\nabla f(x)+\mu Q_{R} x & \text { for } B_{Q} .
\end{array}
$$

(26) is equivalent to (24) when $\mu$ tends to zero (i.e., when we approach the optimal solution), whereas this only happens for (25) when evaluated at current point $(x=\bar{x})$. The $\Theta$ matrices are

$$
\begin{array}{ll}
\Theta=\quad\left(Z X^{-1}+W S^{-1}+\nabla^{2} f(x)\right)^{-1} & \text { for } B, \\
\Theta=\left(Q_{P}+Z X^{-1}+W S^{-1}+\nabla^{2} f(x)\right)^{-1} & \text { for } B_{P}, \text { and } \\
\Theta=\left(\mu Q_{R}+Z X^{-1}+W S^{-1}+\nabla^{2} f(x)\right)^{-1} & \text { for } B_{Q} .
\end{array}
$$

The main difference between (28) and (29) is that $\mu Q_{R}$ tends to zero with $\mu$ and therefore (29) approximates (27) better than (28). Therefore, in general, $B_{Q}$ should be preferred to $B_{P}$. The computational results of this paper have been obtained with $B_{Q}$.

\subsection{Estimating the spectral radius}

Although knowing the spectral radius $\rho$ of $D^{-1}\left(C^{\top} B^{-1} C\right)$ would be instrumental to forecast the efficiency of the preconditioner, its computation is impractical. Even computing its upper bound (19) may be prohibitive, but for some particular classes of problems.

However, a procedure to estimate $\rho$ was recently introduced in [9] for $\phi=0$, i.e., when the preconditioner $M^{-1}$ only includes one term of the power series, thus being equal to $D^{-1}$. In this case, the preconditioned system $\left(D-C^{\top} B^{-1} C\right) \Delta \lambda_{2}=\bar{g}$ solved by PCG in asymmetric form is

$$
\left(I-D^{-1}\left(C^{\top} B^{-1} C\right)\right) \Delta \lambda_{2}=D^{-1} \bar{g}
$$

Clearly, by linear algebra, if $\sigma_{\min }$ is the minimum eigenvalue of $I-D^{-1}\left(C^{\top} B^{-1} C\right)$ then $1-\sigma_{\text {min }}$ is the spectral radius of $D^{-1}\left(C^{\top} B^{-1} C\right)$. The minimum eigenvalue $\sigma_{\min }$ of $I-$ 
$D^{-1}\left(C^{\top} B^{-1} C\right)$ can be estimated from the solution of (10) by PCG, using the relation between PCG and Lanczos method [25]. From this relation (see [23, Chapter 9], 27] for details) it is known that the eigenvalues of the tridiagonal matrices

$$
T_{k}=\left[\begin{array}{ccccc}
\gamma_{1} & \eta_{2} & & & \\
\eta_{2} & \gamma_{2} & \eta_{3} & & \\
& \ddots & \ddots & \ddots & \\
& & \eta_{k-1} & \gamma_{k-1} & \eta_{k} \\
& & & \eta_{k} & \gamma_{k}
\end{array}\right]
$$

$k=1, \ldots, l$, converge to the eigenvalues of the preconditioned matrix of the system solved by PCG as $k$ (the number of PCG iterations) approaches $l$, where the coefficients $\gamma_{i}$ and $\eta_{j}$ can be computed from the PCG algorithm of Figure 1 as follows:

$$
\gamma_{k}=\frac{1}{\alpha_{k-1}}+\frac{\beta_{k-1}}{\alpha_{k-2}}, \quad \beta_{0}=0, \quad \alpha_{-1}=0, \quad \eta_{k+1}=-\frac{\sqrt{\beta_{k}}}{\alpha_{k-1}} .
$$

Eigenvalues of $T_{k}$ are known as Ritz values. In general, the extreme eigenvalues of the preconditioned matrix (the ones we are interested in, for the estimation of the spectral radius) are well approximated already during early PCG iterations [27]. In [9] it was observed that very tight estimations require PCG solutions of high precision.

The previous estimations have been extended in this work to consider any number $\phi \geq 0$ of terms in the power series preconditioner:

Proposition 3.5 Let $M^{-1}=\left(\sum_{i=0}^{\phi}\left(D^{-1}\left(C^{\top} B^{-1} C\right)\right)^{i}\right) D^{-1}$ be the preconditioner with $\phi$ terms of the power series (18). And let $\sigma_{\min }$ be the smallest eigenvalue of the preconditioned matrix $M^{-1} S$. Then the spectral radius of $\left(D^{-1}\left(C^{\top} B^{-1} C\right)\right)$ is

$$
\rho=\sqrt[\phi+1]{1-\sigma_{\min }}
$$

Proof. Denoting $D^{-1}\left(C^{\top} B^{-1} C\right)$ as $Q$, we have

$$
M^{-1} S=\left(I+Q+\cdots+Q^{\phi}\right) D^{-1}(D-Q)=I-Q^{\phi+1} .
$$

By linear algebra, if $\sigma$ is an eigenvalue of $I-Q^{\phi+1}$ with eigenvector $v$, then $\sqrt[\phi+1]{1-\sigma}$ is eigenvalue of $Q$ with the same eigenvector. The spectral radius of $Q$ is thus provided by the smallest eigenvalue $\sigma_{\min }$.

By the previous proposition, $\rho$ can be easily estimated as $\sqrt[\phi+1]{1-\tilde{\sigma}_{\min }}$, where $\tilde{\sigma}_{\min }$ is the smallest Ritz value.

\section{Solver implementation details}

The specialized block-angular IPM described in the previous section has been efficiently implemented in a software package named BlockIP. BlockIP is written in $\mathrm{C}++$, using the object oriented paradigm. It is roughly about 14000 lines of source code, aside from the external package for Cholesky factorization. Actually, BlockIP 
is only linked to the Ng-Peyton block sparse Cholesky package [30]; other more recent Cholesky packages can be added in the future, which may likely improve the performance of the solver. The package can be obtained for research purposes from http://www-eio.upc.edu/ j jcastro/BlockIP.html. The distribution contains a reference manual and an example illustrating the use of the package.

Since BlockIP includes the features of the early prototype described in [12], these will be omitted here. Some additional new features of BlockIP are:

(1) BlockIP may handle linear, quadratic and convex linearly constrained block-angular optimization problems. It may deal with either problems in the standard form (4) and in the more general form

$$
\begin{array}{cl}
\min & f(x) \\
\text { s. to } & b_{l} \leq A x \leq b_{r} \\
& l \leq x \leq u
\end{array}
$$

which are internally transformed to the standard form. Since gradients and Hessians are computed in the original space of variables by user functions, the standard form is preferred (particularly for nonlinear problems) to avoid the extra overhead of transforming the current point to the original space of variables for function evaluations.

(2) BlockIP stops at the feasible point of iteration $j$ when the relative gap between the primal and dual objectives, denoted by $p^{j}$ and $d^{j}$, is below some optimality gap. The relative gap is computed as $\left(p^{j}-d^{j}\right) /\left(1+p^{j}\right)$. For linear and quadratic problems $p^{j}$ and $d^{j}$ are

$$
p^{j}=c^{\top} x^{j}+\frac{1}{2} x^{j^{\top}} Q x^{j} \quad d^{j}=b^{\top} \lambda^{j}-u^{\top} w^{j}-\frac{1}{2} x^{j^{\top}} Q x^{j}
$$

while for nonlinear problems they are computed as

$$
p^{j}=f\left(x^{j}\right) \quad d^{j}=\mathcal{L}\left(x^{j}, \lambda^{j}, z^{j}, w^{j}\right)=f\left(x^{j}\right)-\lambda^{j^{\top}}\left(A x^{j}-b\right)-z^{j^{\top}} x^{j}-w^{j^{\top}} s^{j},
$$

$\mathcal{L}$ denoting the Lagrangian function.

(3) BlockIP implements two types of directions: the standard Newton direction which was described in Section 3, and the second order heuristic direction of [26], which requires the solution of two systems (10) with different right-hand-sides. Both directions can be computed by solving the normal equations by either a Cholesky factorization or by the PCG-based approach of Subsection 3.1. In general, the reduction of iterations caused by the second order direction is not worthwhile when using PCG, since, as far as we know, the solution of the first system can not be efficiently used as a warm-start for the second one. Indeed, from the results of [10] this strategy was not successful for multicommodity flows problems.

(4) The solver implements the two types of regularizations presented in Subsection 3.2 . the proximal point and the quadratic regularization. Although both are similar in practice, the quadratic regularization is preferred by the discussion in Subsection 3.2. Following [14], the quadratic regularization matrix $Q_{R}$ of (23) is heuristically updated at each interior-point iteration $i$ as

$$
Q_{R}:=\delta \cdot i \cdot \mu_{i} / \mu_{0} I
$$

where $\mu_{0}$ and $\mu_{i}$ are the barrier parameter at the starting and current point, and $\delta$ is a (usually small) initial regularization value. The product by $i$, the iteration counter, 


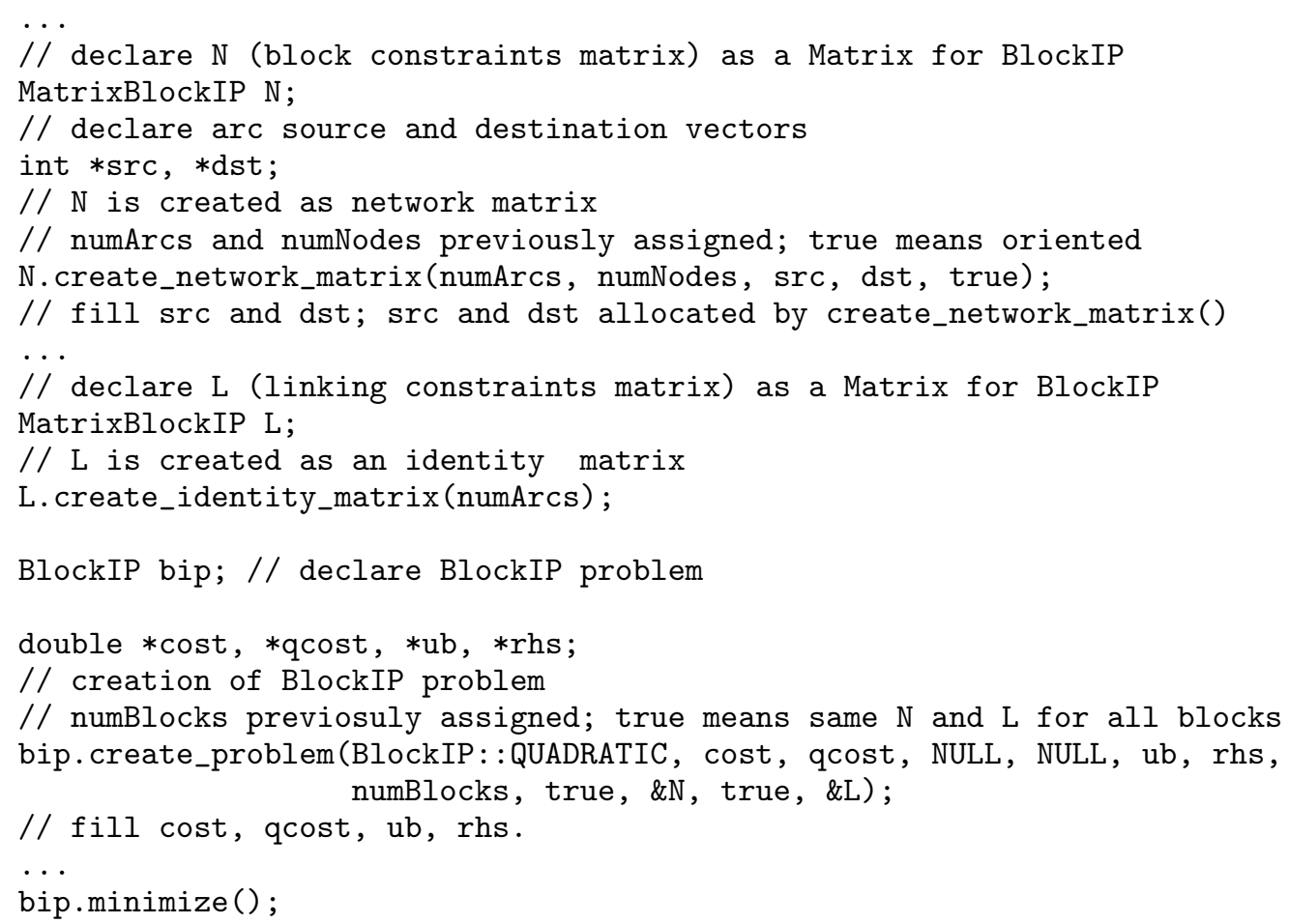

Figure 3. Piece of code illustrating the usage of the BlockIP callable library for the solution of a oriented quadratic multicommodity flow problem

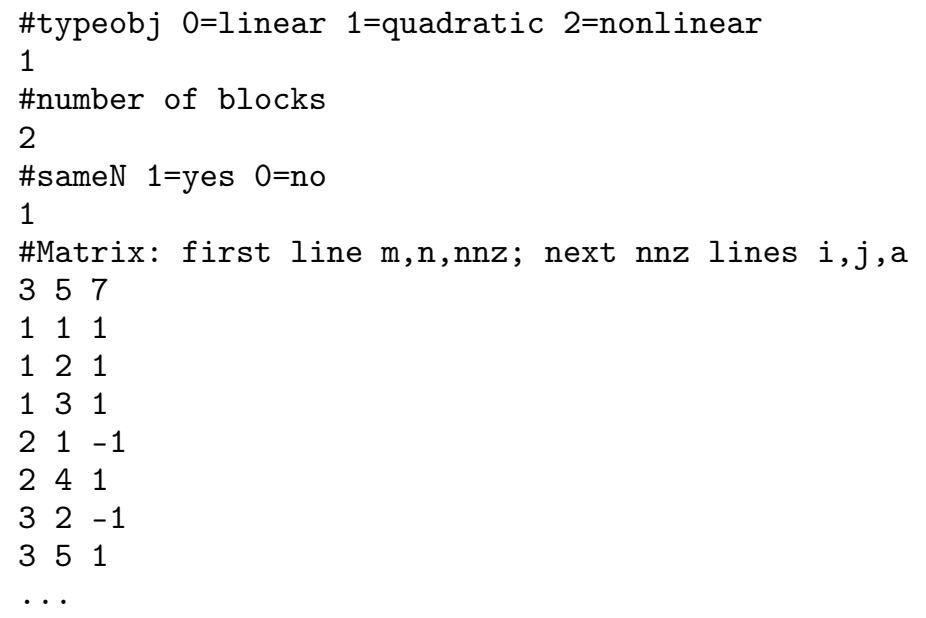

Figure 4. Example using the particular BlockIP file format

is an attempt to compensate for the quick reduction of $\mu_{i}$ when the optimal solution is approached.

(5) BlockIP may compute the Ritz values, and thus the spectral radius $\rho$, for any number $\phi$ of terms in the preconditioner, as described in Subsection 3.3. The value $\phi$ can also be selected by the user. As in [9], Ritz values are efficiently computed by using the SSTEQR LAPACK routine [2]; the extra CPU time needed by this computation is negligible.

(6) Problems can be provided in four different formats.

(a) The most efficient way is using the BlockIP callable library, which provides routines to create problems from matrices and vectors. The code of Figure 3 illustrates how the callable library could be used to formulate, in this example, a oriented quadratic multicommodity flow problem. Particular matrix formats (network - oriented and nonoriented-, general rowwise or columnwise, diagonal, 


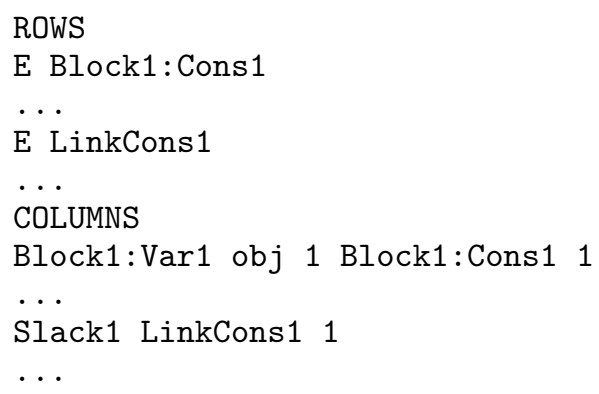

Figure 5. Syntax of row and column entries of structured MPS extension for block-angular problems

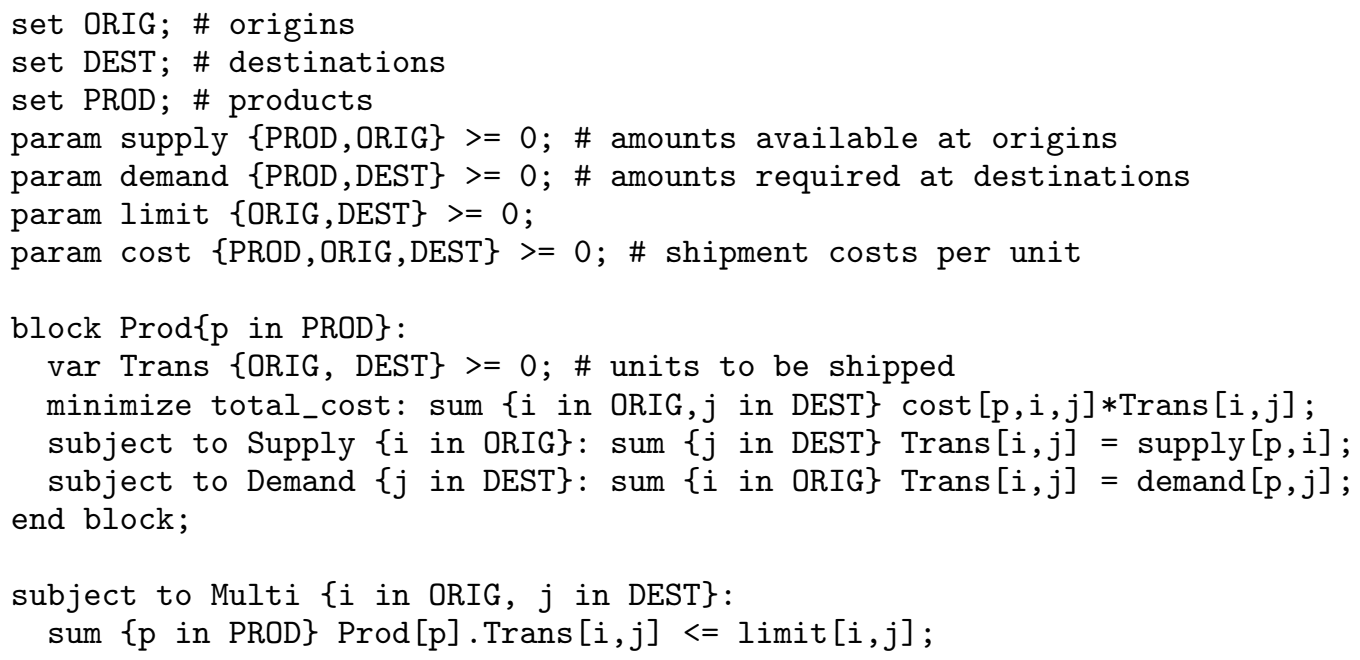

Figure 6. Example of SML-AMPL code for a multicommodity transportation problem

identity, etc.) can be exploited through the callable library. Nonlinear objective functions - including gradient and Hessian evaluations - have to be provided as $\mathrm{C}++$ routines.

(b) Problems can also be efficiently provided by an input file using a specific format for BlockIP. This format consists on a set of scalars, vectors and sparse matrices defining the problem parameters. Sparse matrices are provided in coordinate scheme, i.e, a set of triples $\left(i, j, a_{i j}\right)$ where $(i, j)$ denote the row and column location of the nonzero element $a_{i j}$. An example is shown in Figure 4.

(c) Input files can also be in structured MPS format, an extension of the well-known MPS format created for BlockIP. Figure 5 illustrates the syntax of this format. All variables and constraints preceded by the same prefix (ended with ":") are associated to the same block. Constraints and variables without a prefix correspond, respectively, to linking constraints and their slacks. Standard packages can read structured MPS files without modification.

(d) The last format is based on SML [15], a structure-conveying modelling language based on the popular AMPL [17] modeling language. In addition to hooking BlockIP to it, SML was extended to deal with nonlinear separable problems (see [24] for details). Briefly, SML extends AMPL with the block and end block keywords. Variables and constraints defined within these keywords are associated to the same block, whereas those outside correspond to linking constraints and their slacks. Figure [6 illustrates how a simple multicommodity transportation problem from [17] could be formulated with SML. 
Table 1. Dimensions and results for multicommodity instances

\begin{tabular}{|c|c|c|c|c|c|c|c|c|}
\hline \multirow[b]{2}{*}{ Instance } & \multirow[b]{2}{*}{$k$} & \multirow[b]{2}{*}{$m$} & \multirow[b]{2}{*}{$n$} & \multicolumn{3}{|c|}{ BlockIP } & \multicolumn{2}{|c|}{ CPLEX 12.5} \\
\hline & & & & Iter & $\mathrm{CPU}$ & $\overline{P C G}$ & Iter & $\mathrm{CPU}$ \\
\hline tripart1 & 16 & 3294 & 33774 & 51 & 0.8 & 1260 & 19 & 0.3 \\
\hline tripart2 & 16 & 13301 & 135941 & 68 & 10 & 4034 & 17 & 4 \\
\hline tripart3 & 20 & 25541 & 329161 & 78 & 20 & 3363 & 19 & 13 \\
\hline tripart4 & 35 & 38004 & 869814 & 131 & 268 & 20791 & 24 & 34 \\
\hline gridgen 1 & 320 & 329831 & 985191 & 199 & 253 & 4790 & 33 & 883 \\
\hline
\end{tabular}

\section{Computational results with some applications}

Three applications have been considered for testing the performance of BlockIP: the multicommodity flow problem (linear optimization model), the minimum congestion problem (linear optimization model), and a statistical tabular data confidentiality problem (linear, quadratic or nonlinear optimization model). BlockIP has been compared with the stateof-the-art CPLEX 12.5 package for the linear and quadratic instances. Both the simplex and (interior-point) barrier CPLEX algorithms have been tried for all the instances, and the tables of next subsections report the results with the most efficient option for each instance. For the nonlinear problems we only report results with BlockIP, since other nonlinear interior-point packages could not solve them. The optimality gap was set to $10^{-5}$ for both BlockIP and CPLEX, since tighter tolerances may be problematic for a PCG-based IPM. A value $\phi=0$ was considered for the number of terms of the preconditioner. The quadratic regularization (23) has been used for BlockIP. Default options have been used for other CPLEX and BlockIP parameters, unless explicitly stated. All runs were carried out on a Fujitsu Primergy RX300 server with $3.33 \mathrm{GHz}$ Intel Xeon X5680 CPUs with 144 gigabytes of memory, under a GNU/Linux operating system (OpenSuse 11.4), without exploitation of parallelism capabilities.

\section{$5.1 \quad$ Multicommodity problems}

The purpose of the multicommodity problem is to route a set of items (the commodities) at a minimum cost over a capacitated network. This problem is formulated as

$$
\begin{aligned}
& \min \sum_{i=1}^{k} c^{i^{\top}} x^{i} \\
& \text { s. to } N x^{i}=b^{i} \quad i=1, \ldots, k \\
& \quad \sum_{i=1}^{k} x^{i}+s \leq u \\
& \quad 0 \leq x^{i} \leq u^{i} \quad i=1, \ldots, k, \quad 0 \leq s \leq u,
\end{aligned}
$$

where $N$ is a node-arc incidence matrix of $m^{\prime}+1$ nodes (one node is removed to guarantee full row-rank) and $n^{\prime}$ arcs, $b^{i}$ are the vectors of supply-demand at the nodes for each commodity, $s$ are the slacks of the linking constraints, $u^{i}$ are the vectors of arc capacities for each commodity, and $u$ is the vector of arc total capacities for all the commodities. The number of linking constraints is $l=n^{\prime}$. Multicommodity problems match the general block-angular formulation (11) with $A_{i}=N, L_{i}=I$, and $f_{i}\left(x^{i}\right)=c^{i^{\top}} x^{i}$, for $i=1, \ldots, k$. Note that $D$, defined in (12), is diagonal, since $L_{i}$ are identities, and thus step 10 of the PCG algorithm of Figure 1 only involves a diagonal matrix when $\phi=0$.

Several standard classes of multicommodity instances are available from the literature. Many of them are already satisfactorily solved by generic solvers [8]. Therefore, we selected a small set of - small/medium - five instances which are considered difficult in the 
literature [11]. Table 1 reports, for each instance, the number of blocks $(k)$, constraints $(m)$ and variables $(n)$; the number of IPM iterations ("Iter") and CPU time ("CPU") required by BlockIP and CPLEX; and the overall number of PCG iterations ("PCG") performed by BlockIP. The CPU of the faster run is marked in boldface. From 1 we see that BlockIP is only competitive for the most difficult and largest instance.

\subsection{Minimum congestion problems}

The minimum congestion problem (also known as the maximum concurrent flow [7]) is defined on an infeasible nonoriented multicommodity flow problems. Its purpose is to minimize $\|y\|_{\infty}$, where $y$ is the vector of relative increments in arc capacities needed to make the multicommodity flow problem feasible. This problem, with practical applications in telecommunications, has proved to be difficult for simplex algorithms [6].

Denoting by $x^{i^{+}}$and $x^{i^{-}}$the forward and backward flows for each commodity, and considering the extra variable $t \in \mathbb{R}$, it can be formulated as:

$$
\begin{array}{lr}
\min t & \\
\text { s. to } N x^{i^{+}}-N x^{i^{-}}=b^{i} & i=1, \ldots, k \\
\sum_{i=1}^{k}\left(x_{j}^{i^{+}}+x_{j}^{i^{-}}\right)-y_{j} u_{j} \leq 0 & j=1, \ldots, n^{\prime} \\
y_{j}-t \leq 0 & j=1, \ldots, n^{\prime} \\
x^{i^{+}}, x^{i^{-}} \geq 0 & i=1, \ldots, k \\
y_{j} \geq 0 & j=1, \ldots, n^{\prime}
\end{array}
$$

This formulation contains a dense column because of $t$, and thus the matrix $D$ (needed for the preconditioner) becomes very dense. A more appropriate formulation is obtained by considering $t_{i}, i=1, \ldots, n^{\prime}$ for each arc, with the extra constraints $t_{i}=t_{i+1}$. The resulting model is:

$$
\begin{aligned}
& \min t_{1} \\
& \text { s. to } N x^{i^{+}}-N x^{i^{-}}=b^{i} \quad i=1, \ldots, k \\
& \sum_{i=1}^{k}\left(x_{j}^{i^{+}}+x_{j}^{i^{-}}\right)-y_{j} u_{j} \leq 0 j=1, \ldots, n^{\prime} \\
& y_{j}-t_{j} \leq 0 \quad j=1, \ldots, n^{\prime} \\
& t_{j}-t_{j+1}=0 \quad j=1, \ldots, n^{\prime}-1 \\
& x^{i^{+}}, x^{i^{-}} \geq 0 \quad i=1, \ldots, k \\
& y_{j} \geq 0 \quad j=1, \ldots, n^{\prime}
\end{aligned}
$$

Matrix $D$ of the preconditioner for (35) is of larger dimension but sparser. Unlike in Subsection 5.1, the linking constraints, and thus $D$, are not diagonal matrices.

Table 2 reports the dimensions and results for some instances. They were obtained by making infeasible some multicommodity instances (produced with the standard Mnetgen generator) increasing the demands and supplies. The meaning of the columns is the same as in Table 1. The two numbers in the instance name denote the number of arcs and nodes of the network. BlockIP was competitive even for the smaller instances, being up to ten times faster for the larger ones. 
Table 2. Dimensions and results for minimum congestion instances

\begin{tabular}{|c|c|c|c|c|c|c|c|c|}
\hline \multirow[b]{2}{*}{ Instance } & \multirow[b]{2}{*}{$k$} & \multirow[b]{2}{*}{$m$} & \multirow[b]{2}{*}{$n$} & \multicolumn{3}{|c|}{ BlockIP } & \multicolumn{2}{|c|}{ CPLEX 12.5} \\
\hline & & & & Iter & $\mathrm{CPU}$ & $\mathrm{PCG}$ & Iter & $\mathrm{CPU}$ \\
\hline M32-32 & 34 & 2449 & 33533 & 93 & 0.9 & 289 & 17 & 1.3 \\
\hline M64-64 & 66 & 5564 & 67962 & 94 & 2 & 183 & 17 & 4 \\
\hline M128-64 & 66 & 11640 & 155742 & 97 & 7 & 234 & 19 & 22 \\
\hline M128-128 & 130 & 19867 & 314243 & 97 & 15 & 213 & 20 & 52 \\
\hline M256-256 & 258 & 71891 & 1139467 & 110 & 161 & 891 & 22 & 627 \\
\hline M512-64 & 66 & 470075 & 634143 & 131 & 95 & 1223 & 21 & 1071 \\
\hline M512-128 & 130 & 79765 & 1249145 & 131 & 244 & 2090 & 25 & 2520 \\
\hline
\end{tabular}

\subsection{Statistical tabular data confidentiality problems}

National statistical agencies have to guarantee that confidential information can not be be disclosed from tabular data released. One of the data protection approaches consists, broadly speaking, in computing the closest safe table to the original one, in an attempt to minimize the information loss (see, for instance, [13] for a recent survey on these topics). Representing a table of $n^{\prime}$ cells as a vector $a \in \mathbb{R}^{n^{\prime}}$ that satisfies the constraints $A a=b, l_{a} \leq a \leq u_{a}$, the goal is to find cell perturbations $x \in \mathbb{R}^{n^{\prime}}$ such that:

- minimize $\|x\|_{\ell}$ for some distance $\ell$;

- $x+a$ satisfy the table constraints, that is, $A(x+a)=b, l_{a} \leq x+a \leq u_{a}$, or equivalently $A x=0, l \leq x \leq u$, where $l=l_{a}-a$ and $u=u_{a}-a$;

- a subset of sensitive cells $\mathcal{S} \subseteq\left\{1, \ldots, n^{\prime}\right\}$ is safely shifted from their original values, that is, $\alpha_{i} \leq x_{i} \leq \beta_{i} \quad i \in \mathcal{S}$, where $\alpha_{i}, \beta_{i}$ are some given parameters such that $0 \notin\left[\alpha_{i}, \beta_{i}\right]$.

This results in the following optimization problem:

$$
\begin{array}{cl}
\min _{x} & \|x\|_{\ell} \\
\text { s. to } & A x=0 \\
& l \leq x \leq u \\
& \alpha_{i} \leq x_{i} \leq \beta_{i} i \in \mathcal{S},
\end{array}
$$

which exhibits a block-angular structure for some classes of tables, such as threedimensional ones, i.e., boxes of data obtained by crossing three categorical variables (see [13] for details).

Using $\ell_{1}$, and considering the splitting $x=x^{+}-x^{-}, x^{+} \geq 0, x^{-} \geq 0$, the objective function of (36) becomes

$$
\|x\|_{\ell_{1}}=\sum_{i=1}^{n}\left|x_{i}\right|=\sum_{i=1}^{n}\left(x_{i}^{+}+x_{i}^{-}\right)
$$

obtaining a linear optimization problem. For the Euclidean distance $\ell_{2}$, no splitting of variables is necessary, resulting in a quadratic optimization problem of objective

$$
\|x\|_{\ell_{2}}^{2}=\sum_{i=1}^{n} x_{i}^{2} .
$$

By Subsection 3.2, the PCG is expected to be more efficient with the quadratic than the linear objective because of the nonzero Hessian. According to that, we also considered a strictly convex nonlinear approximation of $\ell_{1}$ given by the pseudo-Huber function

$$
\varphi_{\delta}\left(x_{i}\right)=\sqrt{\delta^{2}+x_{i}^{2}}-\delta
$$


Table 3. Dimensions and results for $\ell_{1}$

\begin{tabular}{|c|c|c|c|c|c|c|c|c|}
\hline \multirow[b]{2}{*}{ Instance } & \multirow[b]{2}{*}{$k$} & \multirow[b]{2}{*}{$m$} & \multirow[b]{2}{*}{$n$} & \multicolumn{3}{|c|}{ BlockIP } & \multicolumn{2}{|c|}{ CPLEX 12.5} \\
\hline & & & & Iter & CPU & PCG & Iter & $\mathrm{CPU}$ \\
\hline $25-25-25$ & 25 & 1850 & 31875 & 168 & 4 & 16475 & 13 & 1 \\
\hline $25-25-50$ & 50 & 3075 & 63125 & 172 & 12 & 22430 & 14 & 2 \\
\hline $25-50-25$ & 25 & 3100 & 63750 & 194 & 19 & 34863 & 13 & 2 \\
\hline $25-50-50$ & 50 & 4950 & 126250 & 200 & 61 & 57641 & 15 & 10 \\
\hline $50-25-25$ & 25 & 3100 & 63750 & 200 & 28 & 53667 & 14 & 1 \\
\hline $50-25-50$ & 50 & 4950 & 126250 & 62 & 1 & 526 & 15 & 7 \\
\hline $50-50-25$ & 25 & 4975 & 127500 & 187 & 33 & 28669 & 15 & 9 \\
\hline $50-50-50$ & 50 & 7450 & 252500 & 133 & 16 & 5523 & 16 & 41 \\
\hline $100-100-100$ & 100 & 29900 & 2010000 & 23 & 8 & 25 & 8 & 986 \\
\hline $100-100-200$ & 200 & 49800 & 4010000 & 32 & 25 & 35 & 9 & 2262 \\
\hline $200-100-200$ & 200 & 79800 & 8020000 & 32 & 49 & 42 & 10 & 8789 \\
\hline $200-200-200$ & 200 & 119800 & 16040000 & 35 & 144 & 49 & 8 & 64521 \\
\hline $500-500-50$ & 50 & 299950 & 25250000 & 40 & 424 & 57 & 9 & 19595 \\
\hline $500-50-500$ & 500 & 299500 & 25025000 & 54 & 227 & 76 & 9 & 17415 \\
\hline
\end{tabular}

Table 4. Dimensions and results for $\ell_{2}$

\begin{tabular}{|c|c|c|c|c|c|c|c|c|}
\hline \multirow[b]{2}{*}{ Instance } & \multirow[b]{2}{*}{$k$} & \multirow[b]{2}{*}{$m$} & \multirow[b]{2}{*}{$n$} & \multicolumn{3}{|c|}{ BlockIP } & \multicolumn{2}{|c|}{ CPLEX 12.5} \\
\hline & & & & Iter & $\mathrm{CPU}$ & $\overline{\mathrm{PCG}}$ & Iter & $\mathrm{CPU}$ \\
\hline $25-25-25$ & 25 & 1850 & 16250 & 11 & 0.0 & 22 & 9 & 0.8 \\
\hline $25-25-50$ & 50 & 3075 & 31875 & 10 & 0.1 & 13 & 9 & 1.4 \\
\hline $25-50-25$ & 25 & 3100 & 32500 & 10 & 0.1 & 14 & 8 & 1.2 \\
\hline $25-50-50$ & 50 & 4950 & 63750 & 10 & 0.1 & 12 & 7 & 5.8 \\
\hline $50-25-25$ & 25 & 3100 & 32500 & 10 & 0.1 & 15 & 9 & 1.2 \\
\hline $50-25-50$ & 50 & 4950 & 63750 & 10 & 0.1 & 13 & 8 & 4.2 \\
\hline $50-50-25$ & 25 & 4975 & 65000 & 10 & 0.1 & 12 & 8 & 5.1 \\
\hline $50-50-50$ & 50 & 7450 & 127500 & 10 & 0.2 & 12 & 7 & 19 \\
\hline $100-100-100$ & 100 & 29900 & 1010000 & 10 & 3 & 10 & 7 & 874 \\
\hline $100-100-200$ & 200 & 49800 & 2010000 & 10 & 6 & 10 & 7 & 1802 \\
\hline $200-100-200$ & 200 & 79800 & 4020000 & 10 & 11 & 10 & 8 & 7319 \\
\hline $200-200-200$ & 200 & 119800 & 8040000 & 9 & 29 & 9 & 8 & 65467 \\
\hline $500-500-50$ & 50 & 299950 & 12750000 & 10 & 91 & 10 & 7 & 15437 \\
\hline $500-50-500$ & 500 & 299500 & 12525000 & 10 & 28 & 10 & 8 & 14784 \\
\hline
\end{tabular}

Table 5. Dimensions and results for pseudo-Huber function and $\ell_{1}$ in small instances

\begin{tabular}{|c|c|c|c|c|c|c|c|c|}
\hline \multirow[b]{2}{*}{ Instance } & \multirow[b]{2}{*}{$k$} & \multirow[b]{2}{*}{$m$} & \multirow[b]{2}{*}{$n$} & \multicolumn{3}{|c|}{$\varphi_{\delta}$} & \multicolumn{2}{|c|}{$\ell_{1}$} \\
\hline & & & & Iter & $\mathrm{CPU}$ & $\mathrm{PCG}$ & $\mathrm{CPU}$ & PCG \\
\hline $25-25-25$ & 25 & 1850 & 16250 & 156 & 1 & 3285 & 4 & 16475 \\
\hline $25-25-50$ & 50 & 3075 & 31875 & 152 & 2 & 2940 & 12 & 22430 \\
\hline $25-50-25$ & 25 & 3100 & 32500 & 146 & 2 & 2525 & 19 & 34863 \\
\hline $25-50-50$ & 50 & 4950 & 63750 & 159 & 5 & 4658 & 61 & 57641 \\
\hline $50-25-25$ & 25 & 3100 & 32500 & 150 & 2 & 2404 & 28 & 53667 \\
\hline $50-25-50$ & 50 & 4950 & 63750 & 143 & 4 & 4392 & 1 & 526 \\
\hline $50-50-25$ & 25 & 4975 & 65000 & 163 & 4 & 3298 & 33 & 28669 \\
\hline $50-50-50$ & 50 & 7450 & 127500 & 152 & 6 & 1831 & 16 & 5523 \\
\hline
\end{tabular}

$\delta$ being a small positive value. Properties of the pseudo-Huber function and its application to compressed sensing problems are described in [16]. The objective function of the resulting convex optimization problem is

$$
f(x)=\sum_{i=1}^{n} \varphi_{\delta}\left(x_{i}\right) \approx\|x\|_{\ell_{1}} .
$$

Tables 3, 4 and 5 report the results with, respectively, (37), (38) and (40). We generated two types of instances, small and large, clearly separated in the tables. The name of the instances denote the number of categories of the three categorical variables. The instance generator of three-dimensional tables can be retrieved from http://www-eio.upc.es/ jcastro/CTA_3Dtables.html. Large instances could not be 
solved with the pseudo-Huber function. Other state-of-the-art nonlinear IPM packages could not even solve the small instances with the pseudo-Huber function. The meaning of the columns is the same as in tables of previous subsections. For $\ell_{1}$ we see that BlockIP is far more efficient as the size of the optimization problem becomes really large (millions of variables), being one to two orders of magnitude faster than CPLEX. For $\ell_{2}$ the results are even better, being two to three orders of magnitude faster in the larger instances. The benefit of a nonzero Hessian in the quality of the preconditioner is clearly seen from the number of PCG iterations: it is smaller for $\ell_{2}$ than for $\ell_{1}$. Finally, Table 5 shows the results with the pseudo-Huber function. We see again the advantage of a nonzero Hessian: the nonlinear problem is solved more efficiently than the linear one for all the instances (but one), requiring less PCG iterations.

\section{Conclusions}

The BlockIP implementation of the PCG-based IPM using the power series preconditioner has shown to be a very efficient tool for the solution of some classes of large convex separable block-angular problems. We do not claim such good efficiencies can be observed in all block-angular problems; this depends, among other factors, on the value of the spectral radius of $D^{-1}\left(C^{\top} B^{-1} C\right)$ which is problem dependent. Therefore, the regularization strategies included in BlockIP (either the proximal point or the quadratic terms) may be instrumental for some problems. The estimation of the spectral radius through Ritz values can also be used as a guidance for the suitability of this approach to some particular problem.

Among the further tasks to be done we find: improving the efficiency of the PCG by adaptive selection of $\phi$, the number of terms in the preconditioner, using the estimation of $\rho$ by the Ritz values; adaptive selection of either Newton or second-order directions, according to the quality of the preconditioner at each interior-point iteration; testing other (linear and nonlinear) classes of block-angular problems (e.g., routing problems in telecommunication networks, formulated as nonlinear multicommodity flows); using this approach within a more general framework for the solution of large mixed integer problems with block-angular structure (e.g., [28]); and implementing within BlockIP other type of preconditioners, such as, e.g., the hybrid approach described in [9]. Some of these tasks are already under development.

\section{Acknowledgments}

The interface between BlockIP and SML-AMPL and the extension of SML-AMPL to nonlinear separable problems was implemented by Xavi Jiménez. This work has been supported by grants MTM2012-31440 of the Spanish Ministry of Economy and Competitiveness, and SGR-2014-542 of the Government of Catalonia

\section{References}

[1] A. Altman and J. Gondzio, Regularized symmetric indefinite systems in interior point methods for linear and quadratic optimization, Optimization Methods \& Software 11 (1999), pp. 275-302.

[2] E. Anderson, Z. Bai, C. Bischof, S. Blackford, J. Demmel, J. Dongarra, J. Du Croz, A. Greenbaum, S. Hammarling, A. McKenney and D. Sorensen, LAPACK Users' Guide, Third edition, SIAM, Philadelphia, PA, 1999.

[3] S. Bellavia, J. Gondzio and B. Morini, A matrix-free preconditioner for sparse symmetric positive 
definite systems and least-squares problems, SIAM Journal on Scientific Computing 35 (2013), pp. A192-A211.

[4] M. Benzi, Splittings of symmetric matrices and a question of Ortega, Linear Algebra and its Applications 429 (2008), pp. 2340-2343.

[5] L. Bergamaschi, J. Gondzio and G. Zilli, Preconditioning indefinite systems in interior point methods for optimization, Computational Optimization and Applications 28 (2004), pp. 149-171.

[6] D. Bienstock, Potential Function Methods for Approximately Solving Linear Programming Problems. Theory and Practice, Kluwer: Boston, 2002.

[7] D. Bienstock and O. Raskina, Asymptotic analysis of the flow deviation method for the maximum concurrent flow problem, Mathematical Programming, 91, pp. 479-492, 2002.

[8] R. E. Bixby, Solving real-world linear programs: a decade and more of progress, Operations Research, 50 (2002), pp. 3-15.

[9] S. Bocanegra, J. Castro and A.R.L. Oliveira, Improving an interior-point approach for large blockangular problems by hybrid preconditioners, European Journal of Operational Research 231 (2013), pp. 263-273.

[10] J. Castro, A specialized interior-point algorithm for multicommodity network flows, SIAM Journal on Optimization 10 (2000), pp. 852-877.

[11] J. Castro, Solving difficult multicommodity problems through a specialized interior-point algorithm, Annals of Operations Research, 124 (2003), pp. 35-48.

[12] J. Castro, An interior-point approach for primal block-angular problems, Computational Optimization and Applications 36 (2007), pp. 195-219.

[13] J. Castro, Recent advances in optimization techniques for statistical tabular data protection, European Journal of Operational Research 216 (2012), pp. 257-269.

[14] J. Castro and J. Cuesta, Quadratic regularizations in an interior-point method for primal blockangular problems, Mathematical Programming 130 (2011), pp. 415-445.

[15] M. Colombo, A. Grothey, J. Hogg, K. Woodsend and J. Gondzio, A structure-conveying modelling language for mathematical and stochastic programming, Mathematical Programming Computation, 1 (2009), pp. 223-247.

[16] K. Fountoulakis and J. Gondzio, A second-order method for strongly convex l1-regularization problems, Technical Report ERGO-14-005, School of Mathematics, The University of Edinburgh, 2014.

[17] R. Fourer, D.M. Gay and D.W. Kernighan, AMPL: A Modeling Language for Mathematical Programming, Second edition, Thomson Brooks/Cole, Toronto, Canada, 2003.

[18] A. Frangioni and C. Gentile, New preconditioners for KKT systems of network flow problems, SIAM Journal on Optimization 14 (2004), 894-913.

[19] J. Gondzio, Matrix-free interior point method, Computational Optimization and Applications 51 (2012), pp. 457-480.

[20] J. Gondzio, Convergence analysis of an inexact feasible interior point method for convex quadratic programming, SIAM Journal on Optimization 23 (2013), pp. 1510-1527.

[21] J. Gondzio and R. Sarkissian, Parallel Interior Point Solver for Structured Linear Programs, Mathematical Programming 96 (2003) pp. 561-584.

[22] C. Keller, N.I.M. Gould and A.J. Wathen, Constraint preconditioning for indefinite linear systems, SIAM Journal on Matrix Analysis and Applications 21 (2000), pp. 1300-1317.

[23] G.H. Golub and C.F. Van Loan, Matrix Computations, Third edition, The Johns Hopkins University Press, Baltimore, MA, 1996.

[24] X. Jiménez, A modelling and optimization environment for large-scale block-angular problems (in Catalan), MSc thesis, Barcelona School of Informatics, Universitat Politècnica de Catalunya, 2012.

[25] C. Lanczos, An iteration method for the solution of the eigenvalue problem of linear differential and integral operators, Journal of Research of the National Bureau of Standards 45 (1950), pp.225-280.

[26] S. Mehrotra, On the implementation of a primal-dual interior point method, SIAM Journal on Optimization 2 (1992), pp. 575-601.

[27] G. Meurant, The Lanczos and Conjugate Gradient Algorithms: from Theory to Finite Precision Computations, SIAM, Philadelphia, PA, 1006.

[28] P. Munari and J. Gondzio, Using the primal-dual interior point algorithm within the branch-priceand-cut method, Computers \& Operations Research 40 (2013), pp. 2026-2036.

[29] Y. Nesterov, Introductory Lectures on Convex Optimization: A Basic Course, Kluwer, Boston, MA, 2004.

[30] E. Ng and B.W. Peyton, Block sparse Cholesky algorithms on advanced uniprocessor computers, SIAM Journal on Scientific Computing 14 (1993), pp. 1034-1056.

[31] A.R.L. Oliveira and D.C. Sorensen, A new class of preconditioners for large-scale linear systems from interior point methods for linear programming, Linear Algebra and its Applications 394 (2005), pp. $1-24$. 
[32] J.M. Ortega, Introduction to Parallel and Vector Solutions of Linear Systems, Plenum Press, New York, NY, 1988.

[33] M.G.C. Resende and G. Veiga, An implementation of the dual affine scaling algorithm for minimumcost flow on bipartite uncapacitated networks SIAM Journal on Optimization, 3 (1993), pp. 516-537.

[34] C. Roos, T. Terláky and J.-P. Vial, Interior Point methods for linear optimization, 2nd Ed., Springer, Boston, MA, 2006

[35] R.J. Vanderbei, Linear Programming: Foundations and Extensions, Kluwer, Boston, MA, 1996.

[36] S.J. Wright, Primal-Dual Interior-Point Methods, SIAM, Philadelphia, PA, 1996. 